\title{
Display Order
}

National Cancer Institute

\section{Source}

National Cancer Institute. Display Order. NCI Thesaurus. Code C93555.

The sequence or position of a component in a list. 